\title{
Research Paper \\ Investigating the Effect of Parent Participation on Quality of Life and Adjustment in Students
}

\author{
*Isa Barghi ${ }^{1}\left(\mathbb{0}\right.$, Peyman Garyi Garavand ${ }^{1}$
}

1. Department of Educational, Faculty of Education and Psychology, Azarbaijan Shahid Madani University, Tabriz, Iran.

\begin{tabular}{c|l}
\hline $\begin{array}{l}\text { Use your device to scan } \\
\text { and read the article online }\end{array}$ \\
justment in Students (Persian)]. Journal of School Psychologh and Institutions, 9(4), 96-105. https://doi.org/10.32598/JSPI.9.4.8 \\
dol'https://doi.org/10.32598/JSPI.9.4.8
\end{tabular}

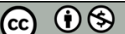

Article Info:

Received: 28 Apr 2018

Accepted: 01 Feb 2019

Available Online: 01 Jan 2021

Key words:

Parental involvement, Quality of academic life, Adjustment

\section{ABSTRACT}

Objective The aim of this study was The effect of parental participation on the quality of academic life and the Compatibility of students.

Methods The research method was descriptive and structural equations. The population of this study was male and female high school students in the fourth district of Tabriz in the academic year 2018-2019, which using Cochran's formula and random sampling method 371 people (172 girls and 119 boys). The instruments used included the Williams and Button quality of life questionnaire, Sinha and Singh adaptation questionnaire, and Fan and Williams academic conflict questionnaire. Data analysis using structural equation statistical test with Smart PLS.2 software was developed.

Results The results showed that there was a positive and significant relationship between parental participation and quality of academic life $(r=96.969, t=10)$ and also between parental participation and student adjustment) ( $r=0.309, t=4)$. The structural equations also show that parental involvement plays a mediating role in students' quality of academic life and adjustment.

Conclusion Therefore, parental involvement can play an essential role in the quality of education and student adjustment.

\section{Extended Abstract}

\section{Introduction}

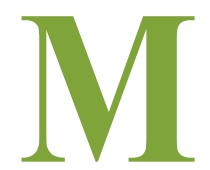

ost adolescents spend much of their time at school (McCoy, George, Cummings, \& Davies, 2013). For students, a school complex is a place not only to learn lessons but also to develop social skills and competencies and improve autonomy, self-adjustment, and self-expression (Parent, Jones, Forehand, Cuellar, \& Shoulberg, 2013). The role of parents is a key factor in understanding how schoolchildren adapt to the needs and con-

\section{* Corresponding Author:}

Isa Barghi, PhD.

Address: Department of Educational, Faculty of Education and Psychology, Azarbaijan Shahid Madani University, Tabriz, Iran.

Tel: +98 (914) 1904108

E-mail: isabarqi@yahoo.com 
sions in the face of issues and use them later in life (Banner et al., 2016). Besides, parents' proper emotional control is one of the most important factors in preventing wrong and inappropriate behaviors at home and in school. Additionally, parent academic engagement, such as engagement with school officials and teachers increases the parental understanding of children's performance (Huang and Gove, 2015). Regarding the above-reviewed studies, the present research seeks to answer the question that whether parent participation significantly affects the quality of educational life and adjustment in students?

\section{Materials and Methods}

This research was cross-sectional in terms of implementation, descriptive applied in terms of time, and correlational in terms of the relationships between independent and dependent variables. The statistical population $(\mathrm{N}=11261)$ included all the male $(n=6042)$ and female $(n=5219)$ high school students in the fourth district of Tabriz City. The Morgan table was used to determine the sample size, so that, using the stratified random sampling method we selected 371 students, including 199 male students and 172 female students. The tools used for this study included the following three categories of questionnaires.

The students' personal-social adjustment questionnaire (Sinha \& Singh, 1993) includes 39 questions assessing the quality of life of students in school. The questions are scored on a 4-point Likert scale from 1 (strongly disagree) to 4 (strongly agree).

The 55-item students' individual-social adjustment questionnaire is scored binary ( 0 and 1$)$. High and low scores in this scale indicate incompatibility and adjustment, respectively. The academic engagement inventory (Fan \& Chen, 2010) is a 5-point Likert type scale, accordingly, it is differently scored. Also, it includes eight subscales of parental enthusiasm, parental involvement in extracurricular activities, parental advice, parent-school communication related to student school issues, school contact with parents, parental participation in school meetings, family regulations, and socioeconomic status.

\section{Results}

Out of 371 participants who answered the questionnaires, 199 students (53.5\%) were male and 172 students (46.5\%) were female.

Table 1 indicates the mean and standard deviation of parent participation, the quality of educational life, and student adaptation. Also, Figure shows the results of the main hypothesis testing with structural equations. It shows the standard coefficient $(\mathrm{R})$ and the proposed causal relationships between parent participation, the quality of educational life, and adjustment. Table 2 reports the standard coefficients and $t$ values. The parents' participation is positively and

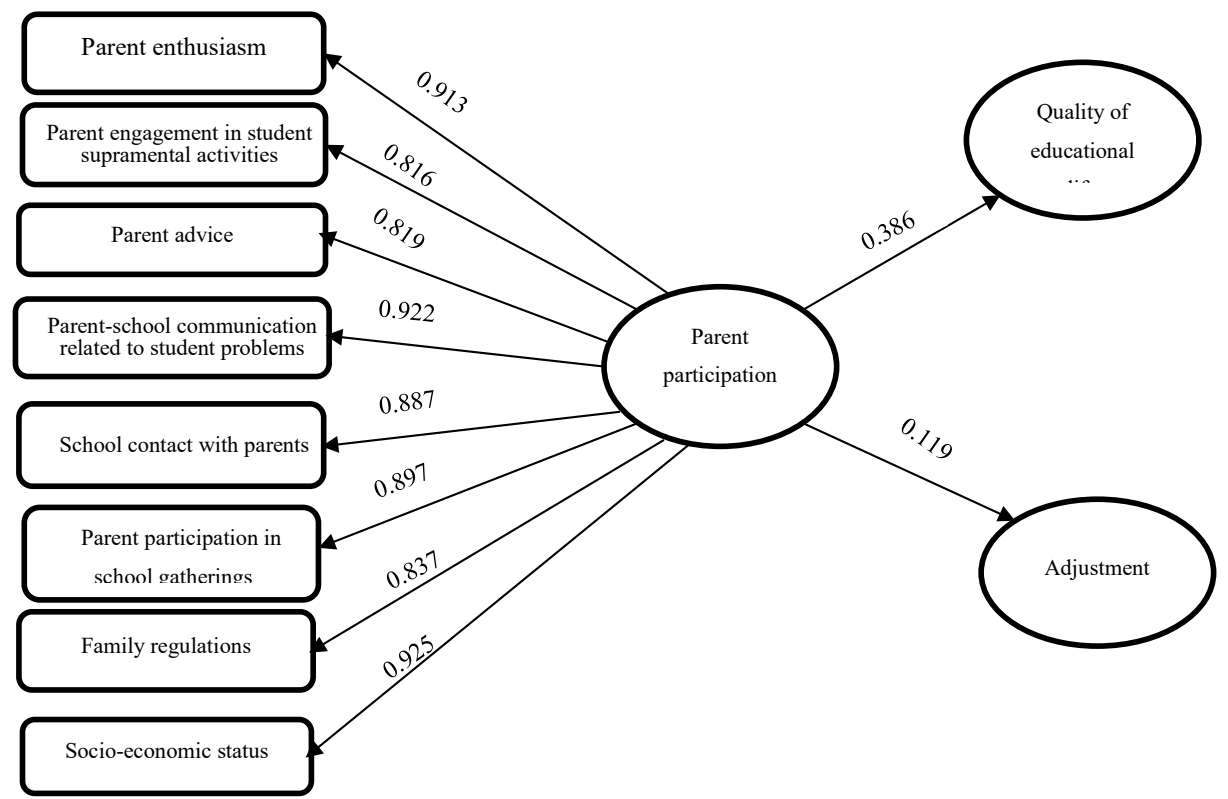

Journal of
School Psychologh and Institutions

Figure 1. A practical model of the effect of parent participation on quality of students' educational life and adjustment in the standard estimation 
Table 1. Mean and standard deviation of the study variables

\begin{tabular}{cc}
\hline Variables & Mean \pm SD \\
\hline Parent participation & $126.19 \pm 10.61$ \\
\hline Quality of educational life & $131.22 \pm 14.86$ \\
\hline Adjustment & $37.93 \pm 5.65$ \\
\hline
\end{tabular}

Table 2. Results of the SEM

\begin{tabular}{cccc}
\hline Research Hypotheses & Standard Coefficient (R) & T & Results \\
\hline Parent participation $\rightarrow$ Quality of educational life & 0.386 & 10.969 & Significant \\
Parent participation $\rightarrow$ Adjustment & 0.119 & 4.307 & Significant \\
\hline
\end{tabular}

significantly correlated with the quality of students' educational life and adjustment.

\section{Discussion and Conclusion}

The research findings showed that parental involvement significantly affected the quality of life and adjustment among students. Consistent with these results, McCoy et al. (2013), Hilland Hofkens (2014), Ratelle, Duchesne, and Guay (2017), Vukovic, Roberts and Green Wright (2013), and Hornby and Blackwell (2018) showed that parental involvement had a significant effect on the quality of life and compatibility. The contribution of parental academic participation is prominent in many educational aspects of students and their outcomes.

According to the Gary Desi and Ryan theory of self-determination (as cited in Vukovic et al., 2013), three categories of supportive behaviors in the field of parent-child relationships explain the adjustment behaviors in school and the quality of students' academic life. According to this theory, the first dimension of parental support is the support of autonomy so that parents consider their child as a unique and independent individual. Through such an attitude, parents behave appropriately to their child's age, identify his/ her needs, and take action to address these needs. The second type of support is participation that involves allocating important resources for the child's learning. Examples of this support include singing up the child in an educational environment with high educational facilities and spending money on educational materials. Finally, the third type of support is the structure that includes a set of behaviors in which parents provide information that the child considers the environment to be predictable, also, parents support their self-regulation and competence (Hornby \& Blackwell, 2018).

\section{Ethical Considerations}

\section{Compliance with ethical guidelines}

There were no ethical considerations to be considered in this research

\section{Funding}

This research did not receive any grant from funding agencies in the public, commercial, or non-profit sectors.

\section{Authors' contributions}

Conceptualization and supervision, investigation, writing - original draft, and writing - review \& editing, funding acquisition and resources: Both authors; Methodology, data collection, data analysis: Isa Barghi.

\section{Conflicts of interest}

The authors declared no conflict of interest. 


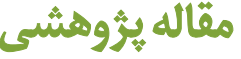

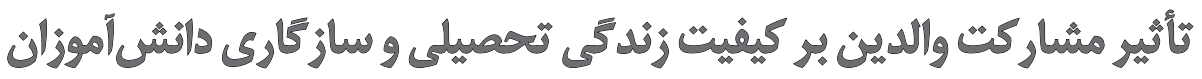 \\ 'عيسى برقى' [ه بيمان كرايى كراوند'}

ا. كروه علوم تربيتى، دانشكده علوم تربيتى و روانشناسى، دانشكَاه شهيد ملنى آذربايجان، تبريز، ايران.

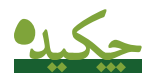

هدف مطالعه حاضر با هدف بررسى تأثير مشاركت والدين بر كيفيت زندكى تحصيلى و سازكارى دانش آموزان انجام شد.

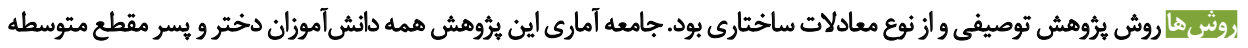

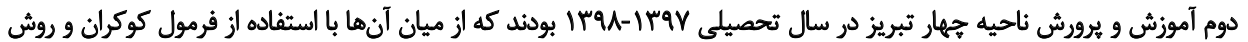

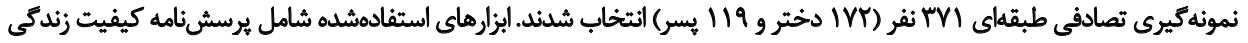

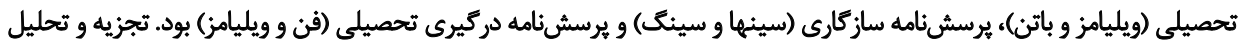

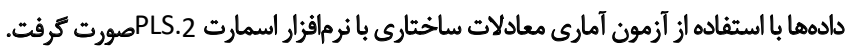

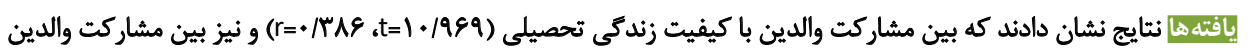

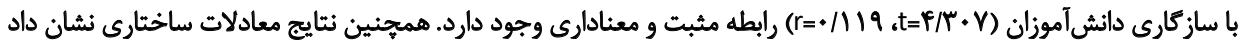

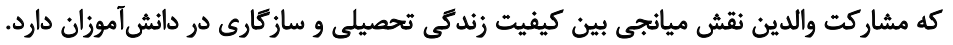

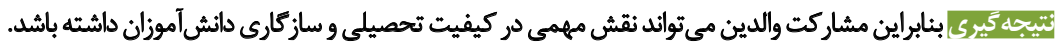

اطلاعات مقاله:

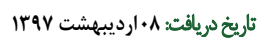

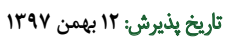

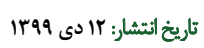

مدرسه، كمك به انجام تكاليف فرزندان، مشاركت در انجمن اولياء

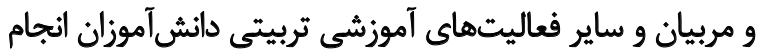

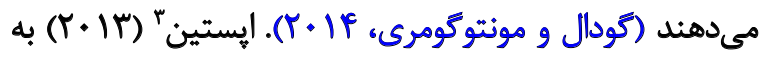

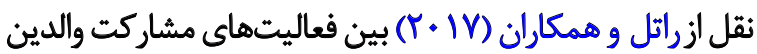

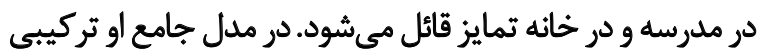

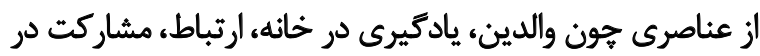

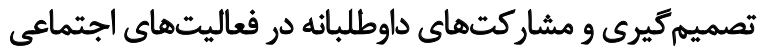

حضور دارند.

يك عامل كليدى براى فهميدن اين موضوع كه دانشآموزان

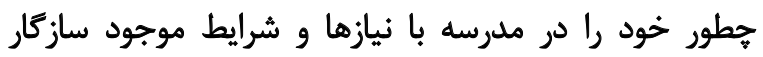

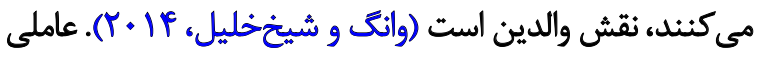

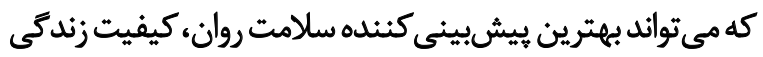

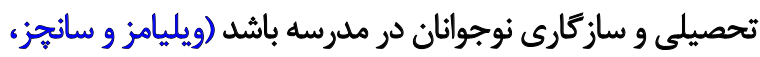

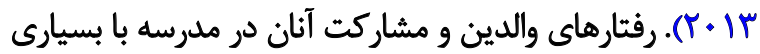

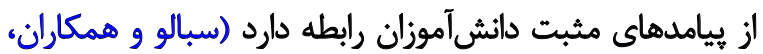

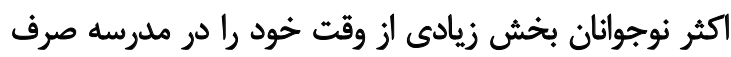

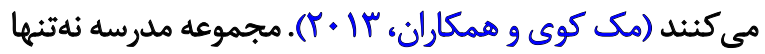

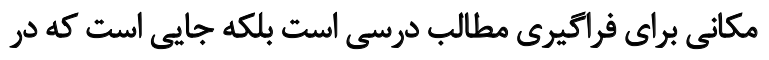

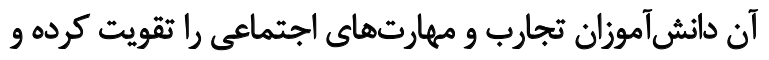

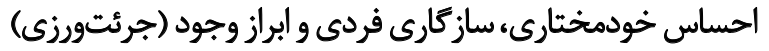

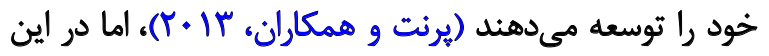

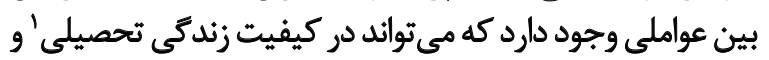

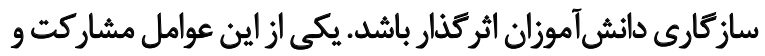

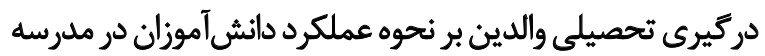

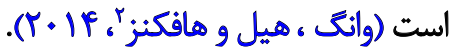
مشاركت والدين مجموعهاى از فعاليتهايى است كه والدين درخصوص يادكيرى فرزندانشان براى مثال شركت در مراسمهاى في اليت 
بوده و از طرفى ديكَر دركيرى و مشاركت والدين مثل تعامل

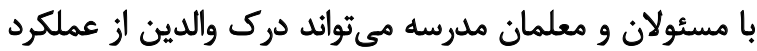

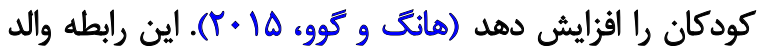

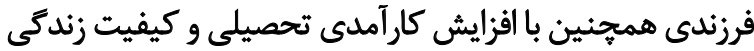

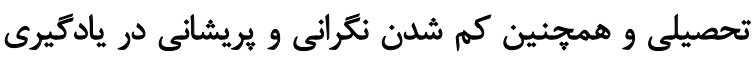

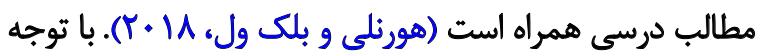

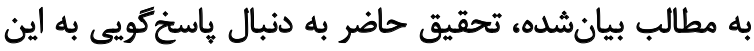

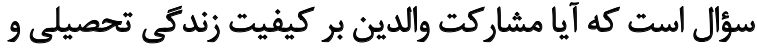

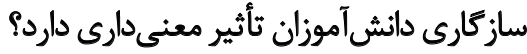

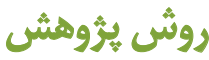

اين يُؤهش از لحاظ معيار اجرايى توصيفى كاربردى، از لحاظ

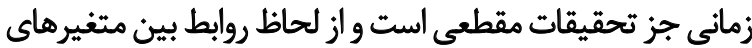
مستقل و وابسته جز تحقيقات همبستئى است.

جامعه، نمونه و روش نمونه

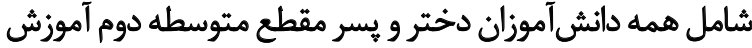

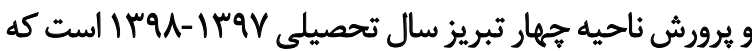
به ترتيب تعداد

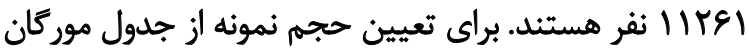

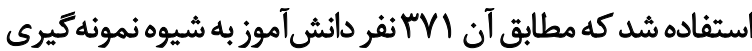

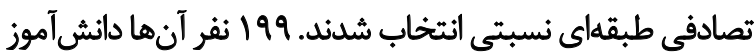

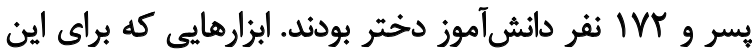

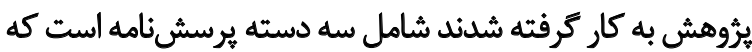
در ادامه نام برده مى كرفوند.

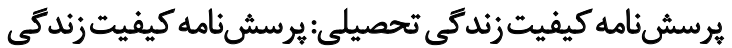

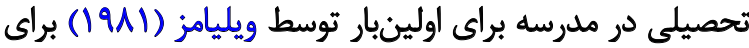

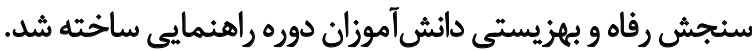

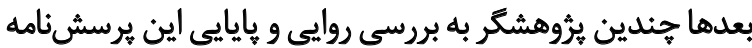

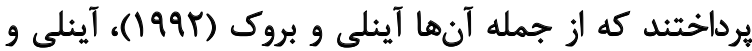

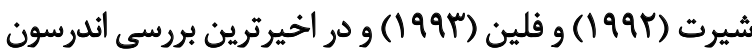

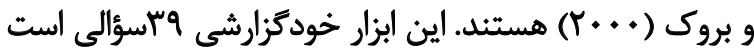

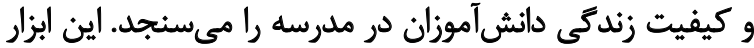

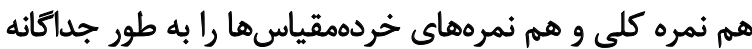

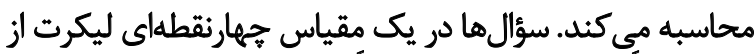

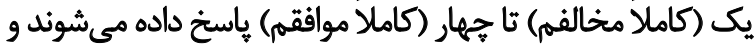

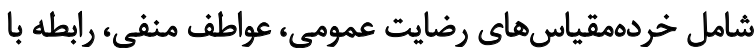

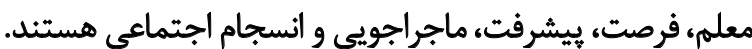

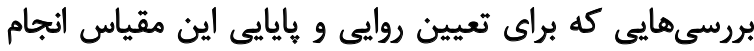
شدهاند، روايى و يايايى آن را تأييد كردهاند.

يرسشنامه سازكارى فردى اجتماعى دانشآموزان: اين

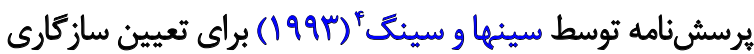

4. Sinha \& Singh

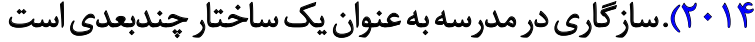

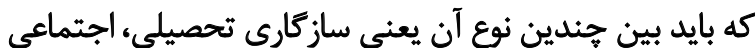

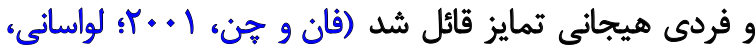

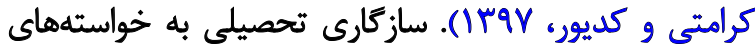

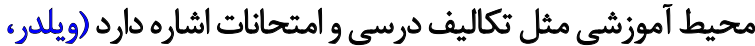

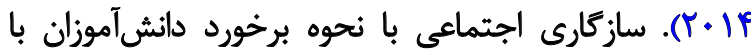

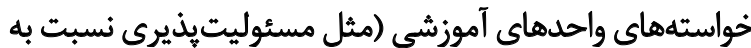

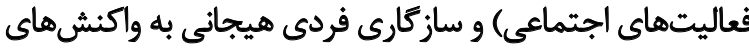

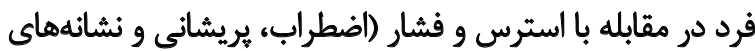

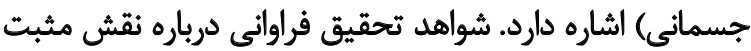

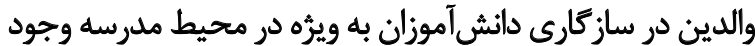

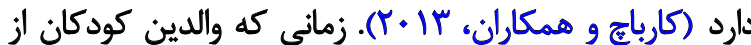

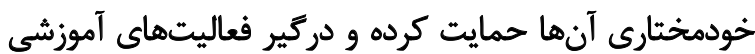

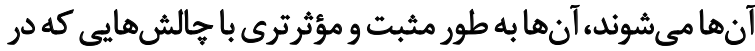

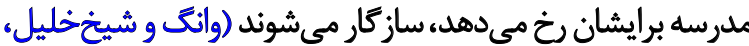

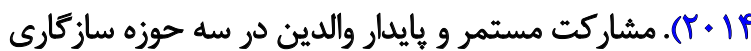

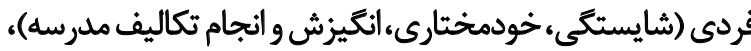

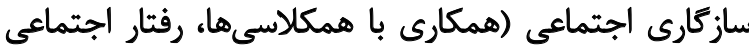

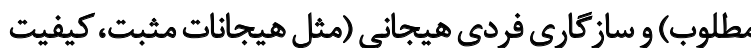

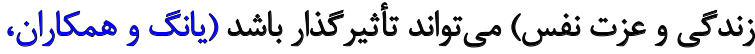

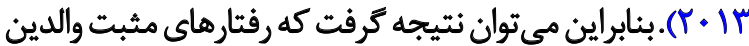

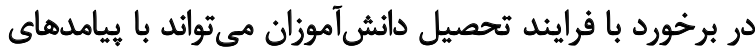
مثبت جون سازكارى تحصيلى دانش آموزان در ارتباط باشئ باشد. همجينين تحقيقات دهلهاى تذشته نشان دادهاند كه درگيرى

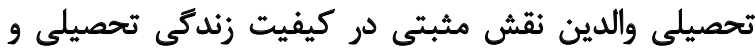

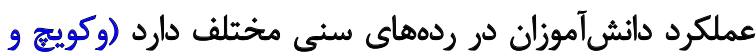

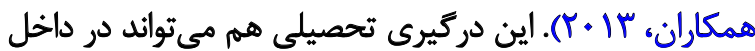

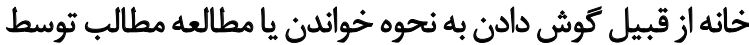

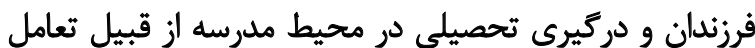

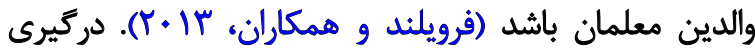

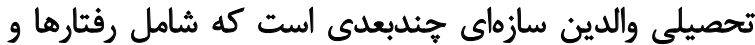

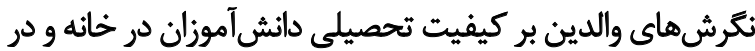

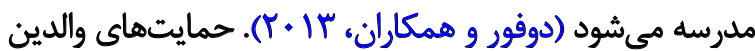

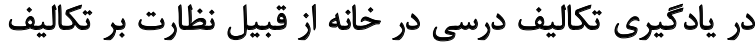

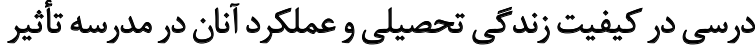

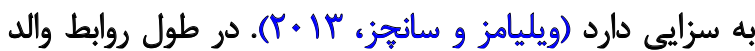

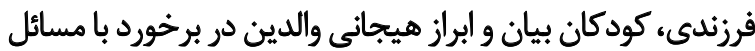

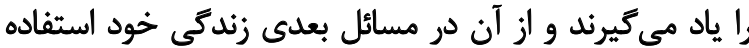

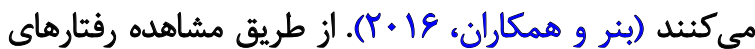

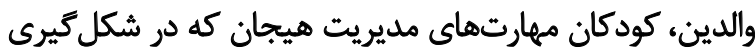

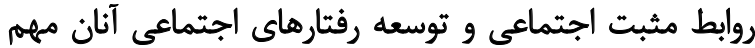

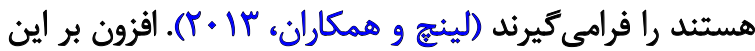

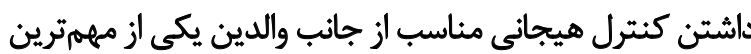

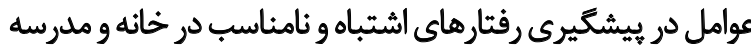




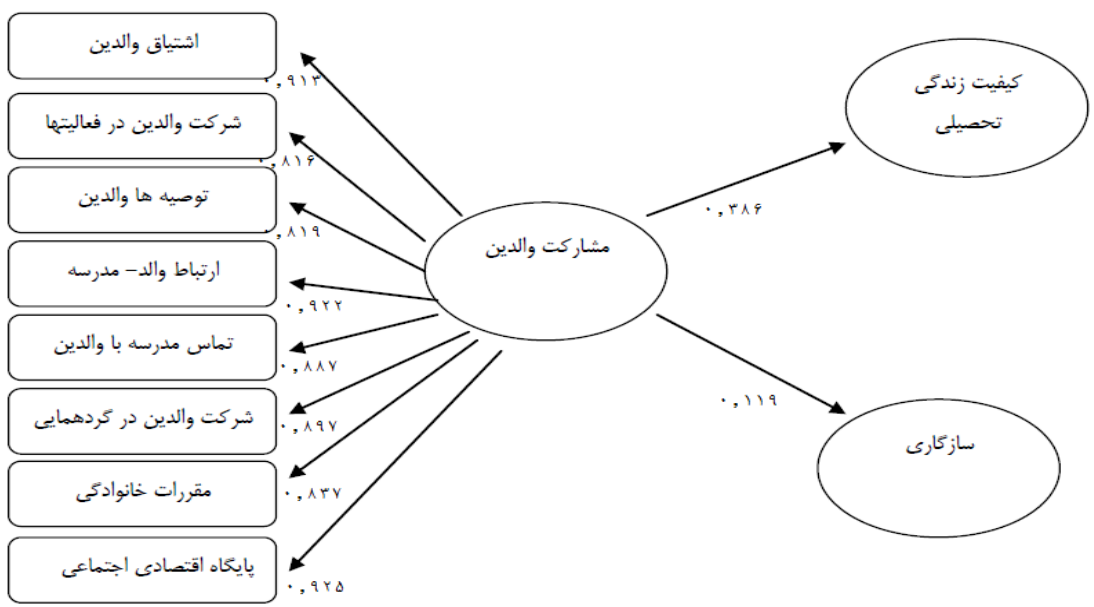

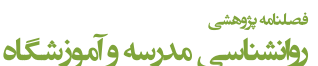
تصوير ا. مدل عملى مشاركت والدين بر كيفيت زندكى تحصيلى و سازكارى دانشآموزان در حالت تخمين استاندارد

والدين در فعاليتهاى فوقبرنامه دانشآموز، شركت والدين در

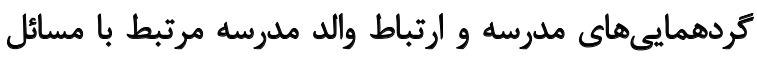

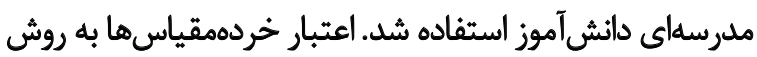

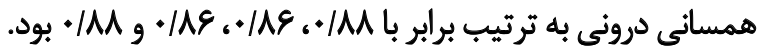

tus

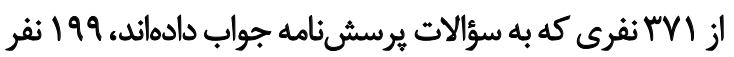

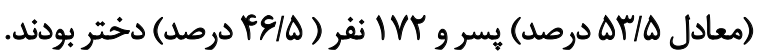

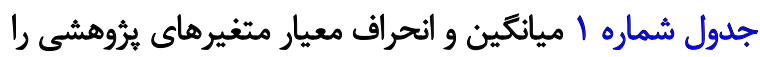

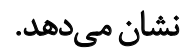

زبراى برآورد رابطه ساختارى تأثير مشاركت والدين بر كيفيت

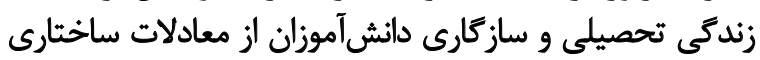

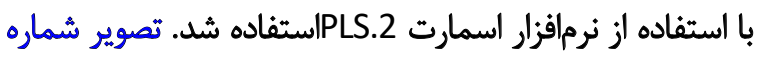

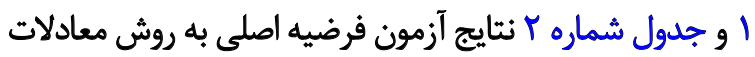

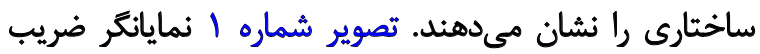

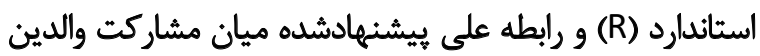

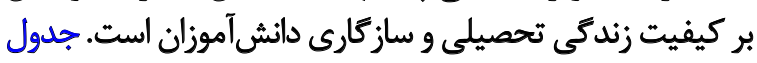

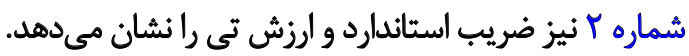
جدول شماره Y نتايج اجراي مدل ساختارى مشاركت والدين

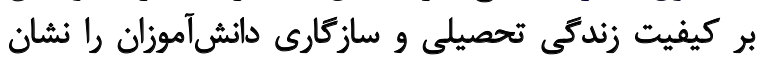

فردى اجتماعى، هيجانى و تحصيلى دانشآموزان ساخته شده است

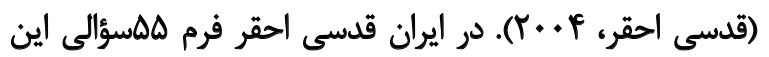

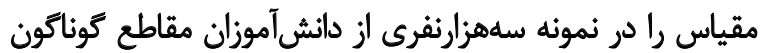

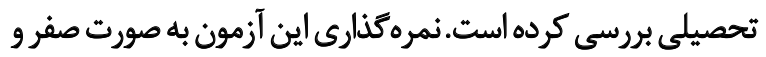

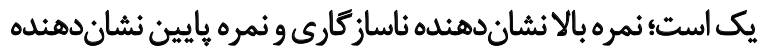

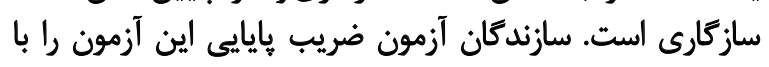

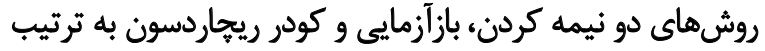

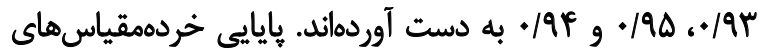

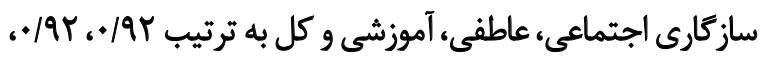

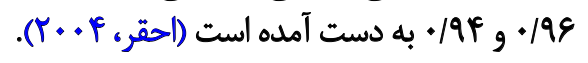

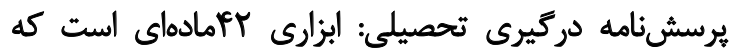

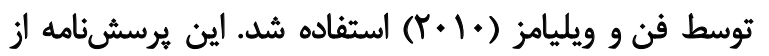

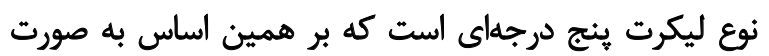

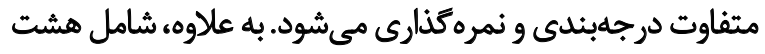

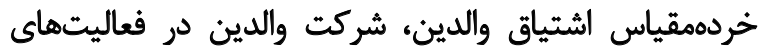

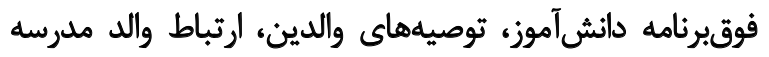

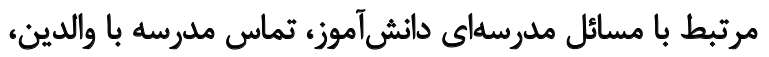

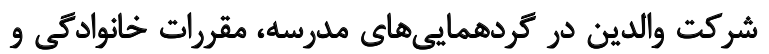

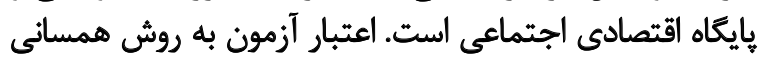

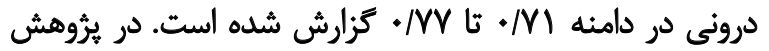

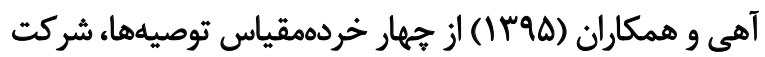

جدول ا. ميانكين، انحراف معيار مشاركت والدين، كيفيت زندكى تحصيلى و سازكارى دانشآموزان

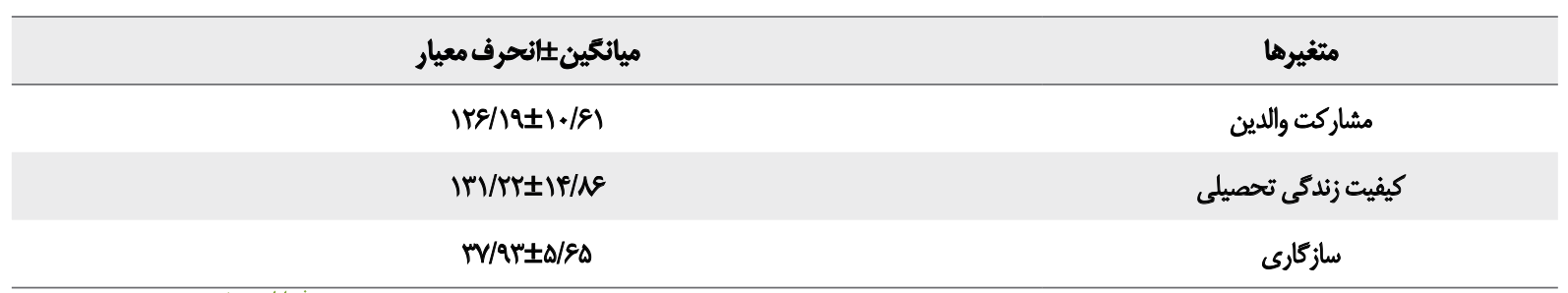

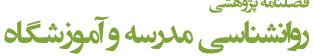


جدول r. نتايج اجراي مدل ساختارى

\begin{tabular}{|c|c|c|c|}
\hline تئيجه & $\mathbf{t}$ & ضريب استاندارد (R) & فر ضيههاى تحقيق \\
\hline 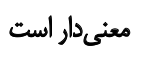 & $1 . / 299$ & . TME & مشاركت والدين † كيفيت زندكى تحصيلى \\
\hline معنى دار است & $p / r \cdot v$ &.$/ 119$ & مشاركت والدين † سازكارى \\
\hline
\end{tabular}

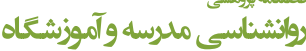

سهم مشاركت تحصيلى والدين در بسيارى از جنبهها

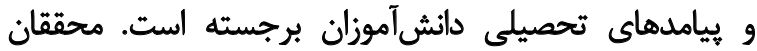

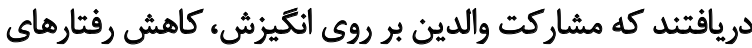

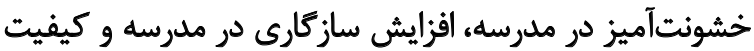

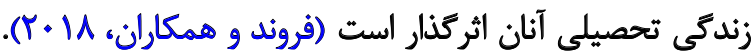

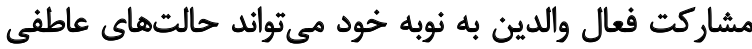

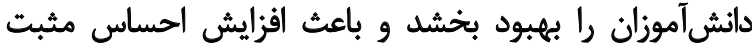

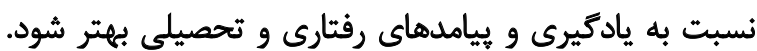

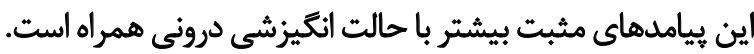

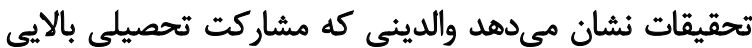

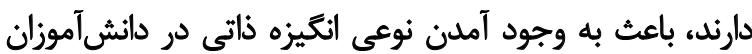

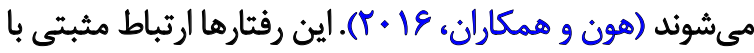

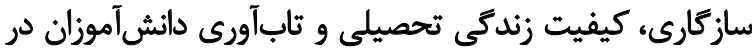

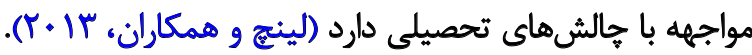

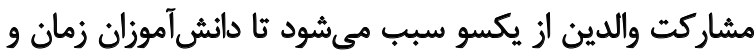

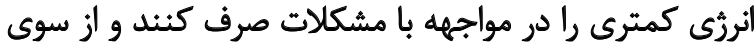

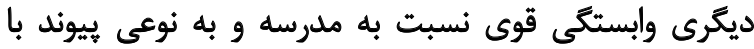

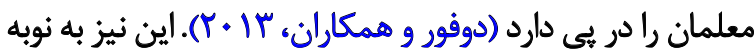

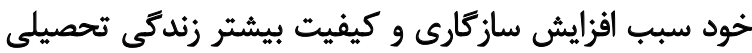

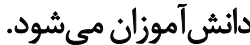

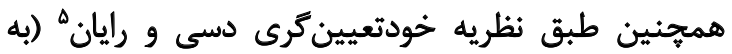

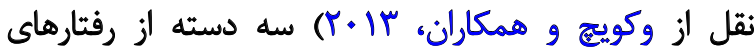

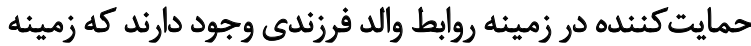

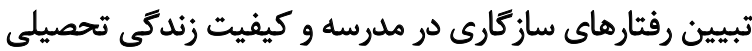

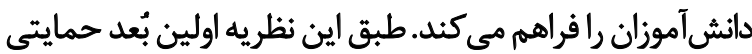

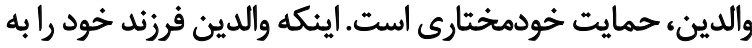

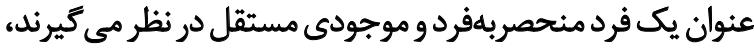

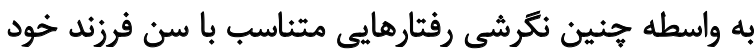

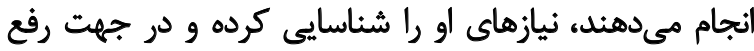

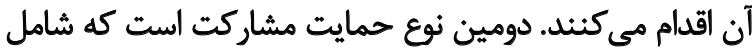

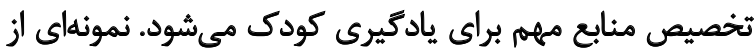

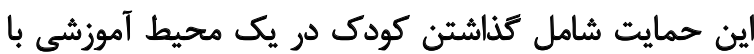

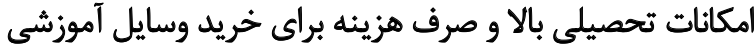

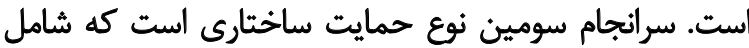

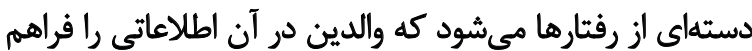

5. Desi and Ryan

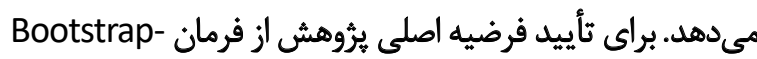

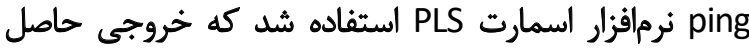

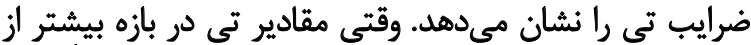

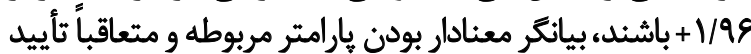

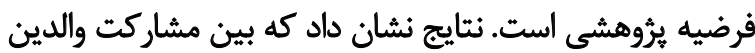

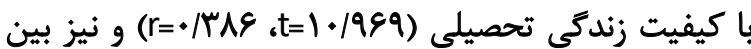

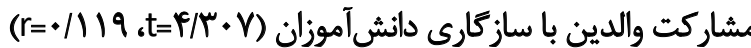
رابطه مثبت و معنى دارى وجود دارد.

بحث و نتيجنه كَيرى

يرثوهش حاضر باهدف بررسى تأثير مشاركت والدين بر كيفيت

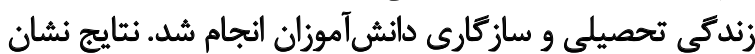

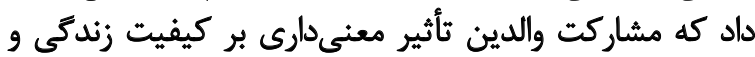

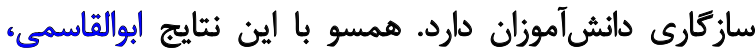

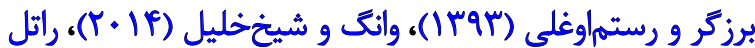

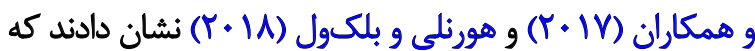

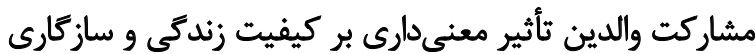

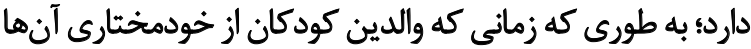

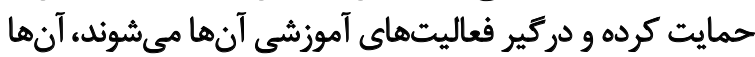

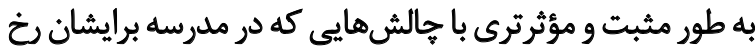

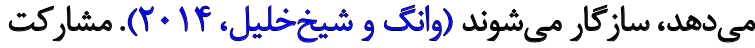

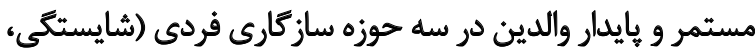

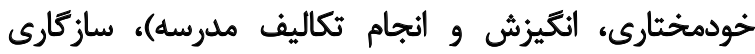

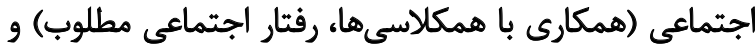

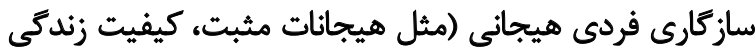

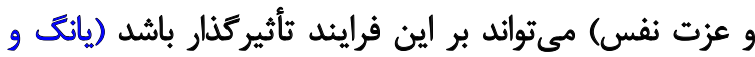

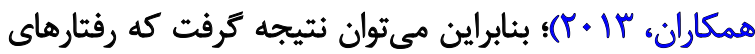

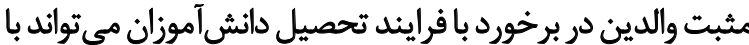

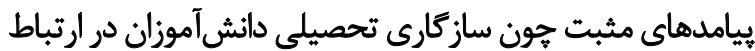

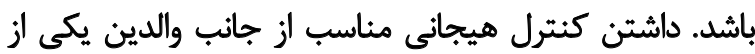

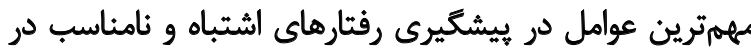

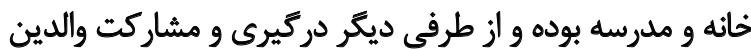

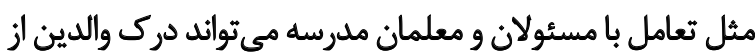

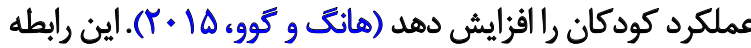

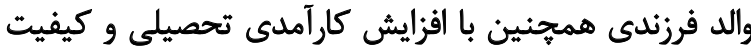

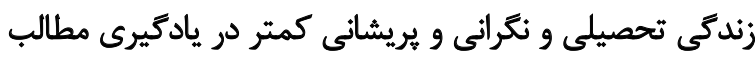

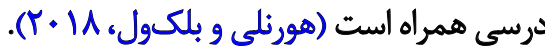


مي كنيد كه كودى محيط اطراف خود را بيشبينىيذير دانسته

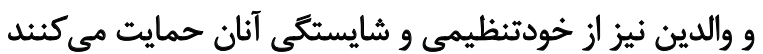

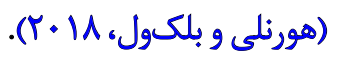

اين يروهش با محدوديتهايى مواجه بود.از آنجايى كه بروهش

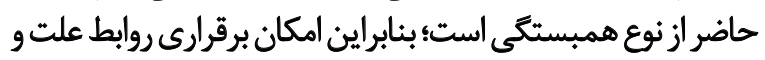

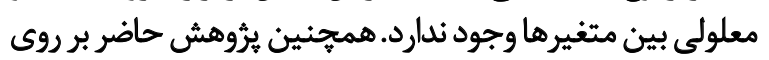

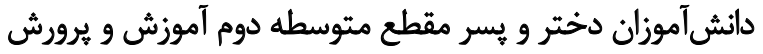

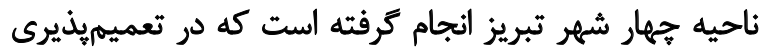

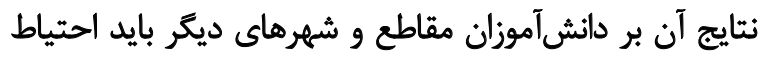

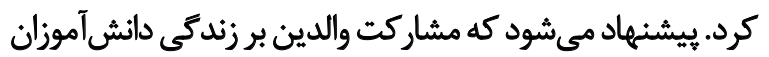

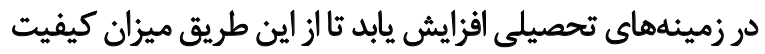

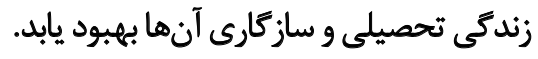

مالاحظاث اخغالاقي

يبروى از اصول اخلاق يثوهش

در اين تحقيق ملاحظات اخلاقى در نظر ترفته نشده است.

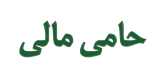

اين تحقيق هيج كونه كمك مالى از سازمان هاى ثأمين مالى در بخش هاى عمومى، تجارى يا غير كون كئفاعى دريافت نكرد.

$$
\text { مشاركت نويسندكًان }
$$

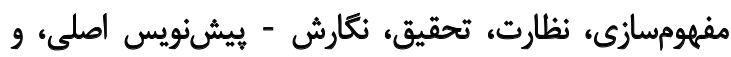

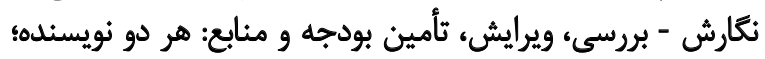

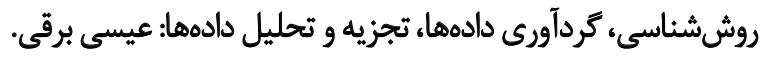

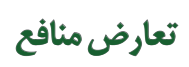

بنابر اظهار نويسندكان اين مقاله تعارض منافع ندارد. 


$$
\text { هئايع فاروسيى }
$$

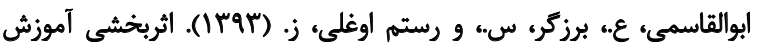

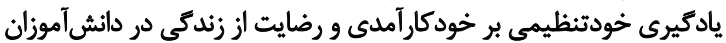

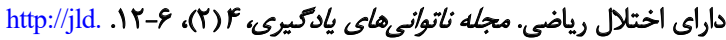
uma.ac.ir/article_258.html

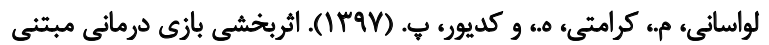

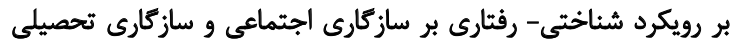

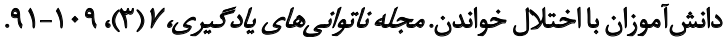
http://jld.uma.ac.ir/article_629_a35798991c11d7cd4785c9eacbf655c1.pdf?lang=en 


\section{References}

Abolghasemi, A., Barzegar, S., \& Rostamoghli, Z. (2015). [The effectiveness of self-regulation learning training on academic motivation and self-efficacy of students with mathematics disorder (Persian)]. Journal of Learning Disabilities, 4(2), 6-21. http://jld.uma.ac.ir/article_258_0.html?lang=en

Benner, A. D., Boyle, A. E., \& Sadler, S. (2016). Parental involvement and adolescents' educational success: The roles of prior achievement and socioeconomic status. Journal of Youth and Adolescence, 45(6), 1053-64. [DOI:10.1007/s10964-016-0431-4] [PMID]

Ceballo, R., Maurizi, L. K., Suarez, G. A., \& Aretakis, M. T. (2014). Gift and sacrifice: Parental involvement in Latino adolescents' education. Cultural Diversity and Ethnic Minority Psychology, $20(1), 116$. [DOI:10.1037/a0033472] [PMID]

Dufur, M. J., Parcel, T. L., \& Troutman, K. P. (2013). Does capital at home matter more than capital at school? Social capital effects on academic achievement. Research in Social Stratification and Mobility, 31, 1-21. [DOI:10.1016/j.rssm.2012.08.002]

Fan, X., \& Chen, M. (2001). Parental involvement and students' academic achievement: A meta-analysis. Educational Psychology Review, 13(1), 1-22. [DOI:10.1023/A:1009048817385]

Fan, W., Williams, C. M. (2010). The effects of parental involvement on students' academic self-efficacy, engagement and intrinsic motivation. Educational Psychology, 30(1), 53-74. https://doi. org/10.1080/01443410903353302

Freund, A., Schaedel, B., Azaiza, F., Boehm, A., \& Lazarowitz, R. H. (2018). Parental involvement among Jewish and Arab parents: Patterns and contextual predictors. Children and Youth Services Review, 85, 194-201. [DOI:10.1016/j.childyouth.2017.12.018]

Froiland, J. M., Peterson, A., \& Davison, M. L. (2013). The longterm effects of early parent involvement and parent expectation in the USA. School Psychology International, 34(1), 33-50. [DOI:10.1177/0143034312454361]

Goodall, J., \& Montgomery, C. (2014). Parental involvement to parental engagement: a continuum. Educational Review, 66(4), 399-410. [DO I:10.1080/00131911.2013.781576]

Hoehn, J., Foxen-Craft, E., Pinder, W., \& Dahlquist, L. M. (2016). The role of parents in promoting children's adjustment to chronic illness. In Child and Adolescent Resilience Within Medical Contexts (pp. 105-119). Cham: Springer. [DOI:10.1007/978-3-319-32223-0 6]

Hornby, G., \& Blackwell, I. (2018). Barriers to parental involvement in education: An update. Educational Review, 70(1), 109-19. [DOI:10.1 080/00131911.2018.1388612]

Huang, G. H. C., \& Gove, M. (2015). Confucianism, Chinese families, and academic achievement: Exploring how confucianism and Asian descendant parenting practices influence children's academic achievement. In Science Education in East Asia (pp. 41-66). Springer, Cham. [DOI:10.1007/978-3-319-16390-1_3]

Jia, R., Kotila, L. E., Schoppe-Sullivan, S. J., \& Kamp Dush, C. M. (2016). New Parents' Psychological Adjustment and Trajectories of Early Parental Involvement. Journal of Marriage and Family, 78(1), 197-211. [DOI:10.1111/jomf.12263] [PMID] [PMCID]

Karbach, J., Gottschling, J., Spengler, M., Hegewald, K., \& Spinath, F. M. (2013). Parental involvement and general cognitive ability as predictors of domain-specific academic achievement in early adolescence. Learning and Instruction, 23, 43-51. [DOI:10.1016/j.learninstruc.2012.09.004]
Lavasani, M., Karamati, M., \& Kadivar, P. (2018). [Effectiveness of cognitive-behavioral play therapy on social adjustment and educational adaptability of students with reading disorder (Persian)]. Journal of Learning Disabilities, 7(3), 91-109. https://www.sid.ir/en/ journal/ViewPaper.aspx?ID=748105

Lynch, A. D., Lerner, R. M., \& Leventhal, T. (2013). Adolescent academic achievement and school engagement: An examination of the role of school-wide peer culture. Journal of Youth and Adolescence, 42(1), 6-19. [DOI:10.1007/s10964-012-9833-0] [PMID]

McCoy, K. P., George, M. R., Cummings, E. M., \& Davies, P. T. (2013) Constructive and destructive marital conflict, parenting, and children's school and social adjustment. Social Development, 22(4), 641-62. [DOI:10.1111/sode.12015] [PMID] [PMCID]

Parent, J., Jones, D. J., Forehand, R., Cuellar, J., \& Shoulberg, E. K. (2013). The role of coparents in African American single-mother families: The indirect effect of coparent identity on youth psychosocial adjustment. Journal of Family Psychology, 27(2), 252. [DOI:10.1037/a0031477] [PMID] [PMCID]

Ahgar, Q. (2004). Standardization of social adjustment questionnaire. Research Plan. [No data] [Link not Found]

Ratelle, C. F., Duchesne, S., \& Guay, F. (2017). Predicting school adjustment from multiple perspectives on parental behaviors. Journal of Adolescence, 54, 60-72. [DOI:10.1016/j.adolescence.2016.11.008] [PMID]

Sinha, E., \& Singh, A. (1993). High School Student Adjustment Questionnaire Guide [A. Karami, Persian trans.]. Tehran: Sina Equip Psychiatric Institute.

Vukovic, R. K., Roberts, S. O., \& Green Wright, L. (2013). From parental involvement to children's mathematical performance: The role of mathematics anxiety. Early Education \& Development, 24(4), 44667. [DOI:10.1080/10409289.2012.693430]

Wang, M. T., Hill, N. E., \& Hofkens, T. (2014). Parental involvemen and African American and European American adolescents' academic, behavioral, and emotional development in secondary school. Child Development, 85(6), 2151-68. [DOI:10.1111/cdev.12284]

Wang, M. T., \& Sheikh-Khalil, S. (2014). Does parental involvement matter for student achievement and mental health in high school? Child Development, 85(2), 610-25. [DOI:10.1111/cdev.12153] [PMID]

Wilder, S. (2014). Effects of parental involvement on academic achievement: A meta-synthesis. Educational Review, 66(3), 377-97. [DOI:10. 1080/00131911.2013.780009]

Williams, T. (1981). The quality of school life. Australia: ERIC. https:// eric.ed.gov/?id=ED208523

Williams, T. T., \& Sánchez, B. (2013). Identifying and decreasing barriers to parent involvement for inner-city parents. Youth \& Society, 45(1), 54-74. [DOI:10.1177/0044118X11409066]

Young, C., Austin, S., \& Growe, R. (2013). Defining parental involvement: Perception of school administrators. Education, 133(3) 291-7. https://www.semanticscholar.org/paper/Defining-ParentalInvolvement $\% 3 \mathrm{~A}$-Perception-of-School-Young-Austin/d325fb2bcf6699408527e842166e722fe8e60f81 